\title{
LAS TRANSICIONES EDUCATIVAS \\ Y SU INFLUENCIA EN EL ALUMNADO
}

\author{
EDUCATIONAL TRANSITIONS \\ AND THEIR INFLUENCE ON STUDENTS
}

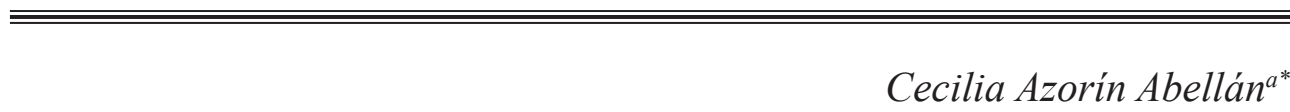

Fechas de recepción y aceptación: 29 de marzo de 2019, 13 de mayo de 2019

Resumen: Las transiciones educativas de una etapa escolar a otra afectan a la población estudiantil en diferentes momentos de su vida académica, provocando cambios en su trayectoria educativa y social, e influyendo notablemente en su futuro. En ocasiones, el impacto de este proceso genera un descenso en el rendimiento del alumnado seguido de episodios de fracaso y abandono escolar. El objetivo de este trabajo es llevar a cabo una revisión de la literatura especializada en las transiciones educativas que permita ahondar en la temática y en sus posibles ámbitos de intervención. Paralelamente, el texto profundiza en la perspectiva holística, el enfoque comunitario y el modelo ecológico-dinámico sobre el que se asienta este proceso. Asimismo, se exponen aspectos susceptibles de interés acerca de las transiciones que suceden desde la Educación Infantil hasta la Educación Secundaria. Por último, se identifican barreras y dificultades, algunas propuestas de mejora a este respecto y futuras vías para avanzar en torno a esta importante línea de conocimiento.

Palabras clave: transición, orientación, escolarización, inclusión, investigación educativa.

Abstract: Educational transitions from one school stage to another affect the student population at different times in their academic life, causing changes in their educational and social trajectory, and notably influencing their future. At times, the impact of this process

${ }^{a}$ Facultad de Educación. Universidad de Murcia.

* Correspondencia: Universidad de Murcia. Facultad de Educación. Departamento de Didáctica y Organización Escolar. Campus de Espinardo, 30100 Murcia. España.

E-mail: cmaria.azorin@um.es 
generates a decrease in student performance followed by episodes of failure and dropout. The aim of this work is to carry out a review of the specialized literature on educational transitions that allows us to delve deeper into the subject and its possible fields of intervention. At the same time, the text examines the holistic perspective, the community approach and the ecological-dynamic model on which this process is based. Likewise, aspects susceptible of interest are exposed on about the transitions that happen from Infant Education to Secondary Education. Finally, barriers and difficulties are identified, as well as some proposals for improvement in this respect and future ways to advance on this important line of knowledge.

Keywords: transition, orientation, schooling, inclusion, educational research.

\section{INTRODUCCIÓN}

Las transiciones educativas son parte de un proceso que está inherentemente ligado a la vida académica del alumnado. Dentro de la literatura especializada, algunas investigaciones recientes en el panorama nacional e internacional han abordado con amplitud este fenómeno y han puesto de manifiesto la necesidad de analizar las transiciones educativas a partir de diferentes vertientes y trayectorias (Álvarez y Rodríguez, 2018; Bereziartua, Zubiri, Intxausti y Odriozola, 2017; Castro, Ezquerra y Argos, 2018; Elias y Daza, 2017; Finnish Ministry of Education and Culture, 2017; Monaheng, 2017; Sánchez y Moreno, 2018; Sierra, 2017; Solís, 2018). Estos estudios muestran el interés que existe en la actualidad por las transiciones educativas desde múltiples perspectivas, por ejemplo: valorando la influencia que este proceso tiene en el éxito del alumnado durante las distintas etapas educativas, su proyección con respecto al mundo laboral, las incidencias que provienen de las escuelas rurales y el impacto de las transiciones en los estudiantes con diversidad funcional o vulnerabilidad. De la lectura de estos textos se deduce que las actuaciones sobre la transición que se planifican en los entornos escolares pueden y deben ser contempladas desde un prisma curricular holístico en el que confluyen diversos agentes educativos y sociales.

Por su parte, Anderson, Jacobs, Schramm y Splittgerber (2000), junto a Gimeno (1996), distinguen los tres grandes momentos del proceso de transición: 1) antes, referido al sujeto y su contexto de procedencia, etapa inmediatamente anterior al cambio; 2) durante, en relación con la transición y aco- 
modación a la nueva situación, y 3 ) después, cuando se logra la estabilización en el nuevo estadio. Por otro lado, Valls (2003) hace mención a cinco fases: 1) preparatoria, 2) de cambio, 3) de asentamiento, 4) de bifurcación y 5) de postransición. Además, existe una serie de factores que influyen en cómo son experimentadas las transiciones. La Organización de los Estados Americanos (2009) diferencia entre: 1) factores internos, que son aquellos relacionados directamente con el alumnado y su entorno inmediato, y 2) factores externos, aquellos vinculados con la instancia educativa a la que se accede.

Otro aspecto que hay que tener en cuenta es el carácter permanente que tienen las transiciones educativas. Para Parrilla y Sierra (2015), el concepto de transición, lejos de ceñirse a los cambios específicos entre etapas educativas, es un proceso permanentemente abierto y presente en la vida educativa de los centros, de las familias y del alumnado. Skouteris, Watson y Lum (2012) coinciden en señalar la transición como un procedimiento clave, tanto individual como colectivo, que no solo se refiere a un momento concreto, sino que se desarrolla a lo largo de la vida personal, social y educativa de sus protagonistas. Asimismo, es conveniente destacar que el fenómeno del traspaso educativo ocurre entre cualquier etapa del sistema escolar, sea o no obligatoria, con similares problemáticas e idénticas estrategias, como la adquisición de información, orientación, coordinación, acogida e integración (Michavila y Esteve, 2011).

Precisamente, la revisión teórica que aquí se presenta permite ahondar en el estado del arte de la transición educativa, que comprende en este caso y de forma acotada las etapas de Educación Infantil, Educación Primaria y Educación Secundaria, desde la atención temprana que tiene lugar en la niñez hasta el fin de la educación obligatoria. Los criterios elegidos para la búsqueda de información sobre la temática objeto de estudio fueron los siguientes: investigaciones empíricas que abordan las transiciones educativas durante las citadas etapas (esto incluye el inicio de la escolaridad -atención temprana- y la culminación de la escolarización obligatoria), estudios teóricos que aportan definiciones sobre el concepto de transiciones -y que en consecuencia, permiten construir conocimiento desde una percepción educativa y social amplia- y experiencias que muestran avances principalmente en el campo de las transiciones escolares y su relación con la visión holística que ofrece el actual modelo comunitario, que tan en boga se encuentra dentro de 
la pedagogía contemporánea. Teniendo en cuenta estos aspectos, las bases de datos consultadas para la localización de las fuentes documentales fueron Dialnet, SciELO, DICE, Scopus y Web of Science, bajo el descriptor común "transiciones educativas" en español e inglés, denominación que da sentido al puzle que se investiga.

El posicionamiento de la transición escolar como línea de indagación preferente ha permitido profundizar en las bases teóricas y evidencias empíricas que aportan conocimiento acerca de este campo de estudio. Actualmente, continúa candente el debate sobre cómo lograr un ejercicio de transición armoniosa, poniendo énfasis en la adaptación, la continuidad y la preparación de la transición, así como en las prácticas docentes que giran en torno a esta temática (Dockett y Perry, 2013). Desde el punto de vista de la orientación, los diferentes momentos de transición escolar que suceden a lo largo del sistema educativo y las actuaciones de acompañamiento dirigidas al alumnado que se programan durante este proceso han ocupado gran parte de los titulares a disposición de la comunidad científica. A este respecto, Fernández y SantosBocero exponen que:

En el contexto escolar, tradicionalmente se han resaltado aquellas transiciones localizadas en momentos peculiares de la incorporación del alumnado a ambientes diferentes a los que se había desenvuelto hasta ese momento, como el paso de la familia a la escuela, de ésta al instituto, de aquí a la universidad y al mundo laboral. Rupturas que implican, en diferentes grados, dificultad añadida a los estudiantes para sostener su ritmo de trabajo académico y relaciones sociales dentro y fuera de las aulas. Procesos que les serán más o menos facilitados en razón, entre otros factores, de cómo el sistema educativo dé continuidad y coherencia al tránsito de uno a otro de dichos ambientes y de las acciones pedagógicas y psicopedagógicas que se pongan en escena para abordarlos (2014: 10).

Con todo, antes de seguir desgranando los principales fundamentos adheridos a las transiciones educativas, el siguiente apartado incluye una breve síntesis sobre el concepto del que parte el artículo para adquirir cierta perspectiva de los distintos matices ofrecidos en estudios previos a la hora de acometer la poliédrica definición sobre las transiciones educativas. 


\section{AproximACIÓN CONCEPTUAL}

Aunque no puede concluirse que exista una definición universalmente aceptada, sí hay cierto consenso en considerar la transición como un fenómeno multifacético que implica una serie de interacciones y procesos a lo largo del tiempo (Dunlop, 2014; Ptriwskyj, Thorpe y Tayler, 2005). Así pues, resulta importante clarificar el término que define el paso entre etapas, tramos, ciclos o niveles dentro del sistema educativo. A continuación, se presenta por orden cronológico un corpus de definiciones que han sido formuladas en la literatura de las últimas tres décadas con la finalidad de arrojar luz acerca del concepto de transiciones. Un hecho que afecta a la vida de las personas no solo desde el punto de vista educativo, sino también social (cuadro 1). En esta dirección, se incluye una perspectiva amplia, con aportaciones generales sobre los procesos de transición, así como otras contribuciones de carácter más específico en el campo de la educación:

\section{CUADRO 1}

Definiciones sobre el significado de transición contemplado desde un punto de vista socioeducativo

\begin{tabular}{|l|l|}
\hline AUTORÍA Y AÑO & \multicolumn{1}{c|}{ DEFINICIÓN } \\
\hline Gimeno (1996) & $\begin{array}{l}\text { "El paso por la vida es el paso por un pasaje cambiante, más o menos } \\
\text { rico, monótono y sorprendente; es un viaje que en parte lo escogemos y } \\
\text { en parte surge y a veces se nos impone..." (p. 78). Por consiguiente, el } \\
\text { término transición se asocia al cambio, supone una alteración en la mane- } \\
\text { ra de ser o de estar y en la forma de hacer una cosa. }\end{array}$ \\
\hline $\begin{array}{l}\text { Corominas e Isus } \\
(1998)\end{array}$ & $\begin{array}{l}\text { "Las situaciones de transición actúan como puentes de interconexión } \\
\text { entre una situación previa y una situación posterior entre las que opera la } \\
\text { adaptación al cambio" (p. 156). Como puede comprobarse, la autoría de } \\
\text { este trabajo contempla el estado o período en que dividimos la vida de } \\
\text { una persona. }\end{array}$ \\
\hline Rogoff (2003) & $\begin{array}{l}\text { Los procesos de transición se consideran experiencias individuales y so- } \\
\text { ciales, construidas activamente a medida que los individuos participan en } \\
\text { procesos sociales y culturales que, por su propia naturaleza, son eventos } \\
\text { comunitarios. }\end{array}$ \\
\hline
\end{tabular}




\begin{tabular}{|c|c|}
\hline AUTORÍA Y AÑO & DEFINICIÓN \\
\hline $\begin{array}{l}\text { Dockett y Perry } \\
(2007)\end{array}$ & $\begin{array}{l}\text { La transición es vista como un conjunto de procesos a medida que los } \\
\text { individuos pasan de un contexto a otro o cambian su rol en las comunida- } \\
\text { des educativas. }\end{array}$ \\
\hline $\begin{array}{l}\text { Fabian y Dunlop } \\
(2007)\end{array}$ & $\begin{array}{l}\text { El concepto de transición educativa hace referencia al cambio que efectúa } \\
\text { el alumnado desde una fase de la educación a otra, en la que se enfrentan } \\
\text { a desafíos desde el punto de vista de las relaciones sociales, el estilo de } \\
\text { enseñanza, el ambiente, el espacio, el tiempo, los contextos de aprendi- } \\
\text { zaje y el propio aprendizaje, de modo que el proceso se caracteriza por } \\
\text { su intensidad y el aumento progresivo de demandas. En definitiva, las } \\
\text { transiciones en el ámbito escolar aluden a un proceso de cambio que ex- } \\
\text { perimenta el alumnado (y sus familias) cuando se mueve de un escenario } \\
\text { a otro, como puede ser de la casa a preescolar. La transición incluye la } \\
\text { extensión de tiempo que toma hacer dicho cambio, desde el inicio del } \\
\text { proceso hasta que el alumnado está totalmente integrado en su nuevo } \\
\text { ambiente. }\end{array}$ \\
\hline Peralta (2007) & $\begin{array}{l}\text { Las transiciones en educación implican procesos de cambio de una estan- } \\
\text { cia inicial a otra por alcanzar; están reguladas culturalmente y transcurren } \\
\text { en el tiempo. Una transición escolar es vista como el paso de una institu- } \\
\text { ción inicial a otra posterior. }\end{array}$ \\
\hline $\begin{array}{l}\text { Vogler, Grivello y } \\
\text { Woodhead (2008) }\end{array}$ & $\begin{array}{l}\text { Las transiciones son eventos claves o procesos que ocurren en momentos } \\
\text { determinados y específicos, y están ligados a cambios en la persona (su } \\
\text { apariencia, actividades, estatus, roles y responsabilidades). En consecuen- } \\
\text { cia, las transiciones involucran ajustes psicosociales y culturales, de modo } \\
\text { que la manera como son vividas depende de la vulnerabilidad o capacidad } \\
\text { de resiliencia que tiene el alumnado. }\end{array}$ \\
\hline Unicef (2012) & $\begin{array}{l}\text { El proceso de tránsito engloba a estudiantes que se trasladan y se adaptan } \\
\text { a nuevos entornos de aprendizaje, familias que aprenden a trabajar dentro } \\
\text { de un entorno sociocultural y centros educativos que admiten a nuevos } \\
\text { estudiantes en su sistema escolar. }\end{array}$ \\
\hline $\begin{array}{l}\text { Fernández y Santos- } \\
\text { Bocero (2014) }\end{array}$ & $\begin{array}{l}\text { "Con el término transiciones nos referimos a los cambios y transfor- } \\
\text { maciones que los seres humanos abordamos a lo largo de la vida en } \\
\text { momentos singulares, más o menos definidos, propiciados por causas o } \\
\text { circunstancias extrínsecas o intrínsecas al individuo, y que conllevan la } \\
\text { necesidad de readaptación a nuevos horizontes, contextos, estados socia- } \\
\text { les, culturales o personales" (p. 9). }\end{array}$ \\
\hline Sebastián (2015) & $\begin{array}{l}\text { Las transiciones son definidas como momentos críticos de cambio que } \\
\text { vive el alumnado al pasar de un ambiente a otro, abriendo oportunidades } \\
\text { para su desarrollo y su aprendizaje para la vida. }\end{array}$ \\
\hline
\end{tabular}


En síntesis, las transiciones son sinónimo de viaje, de travesía. Al hablar de las transiciones en términos de viaje, cabe relacionar este discurso con "el viaje del héroe" que popularizó el mitólogo Joseph Campbell a mediados del siglo pasado, cuya esencia está en connivencia con el viaje hacia la inclusión que proponen Azorín y Ainscow (en prensa), en el que el tránsito de una etapa educativa a otra como foco de interés adquiere especial relevancia. Así pues, es preciso comprender que las transiciones educativas tienen que ver con un proceso cíclico, que se repite a lo largo de la vida académica del alumnado conforme este promociona de una etapa educativa a otra. En consecuencia, es un paso intermedio entre un estado inicial y otro provisional-final que implica adaptarse al medio (sistema educativo) y sobrevivir en él. Para ello, deben tenderse puentes, canales y nexos de unión que permitan derribar fronteras y eliminar barreras entre un mundo y otro, así como construir el andamiaje necesario para recorrer el camino de la manera más gradual y exitosa posible. Esto conlleva tener capacidad de adaptación a situaciones diversas, y mantener la continuidad y coordinación necesaria para que la persona que se encuentra inmersa en dicho proceso pueda progresar en la esfera personal, educativa, social y laboral. En este contexto, según se justifica más adelante, surge la necesidad de desarrollar un enfoque comunitario desde el que responder a los desafíos que plantea el fenómeno de las transiciones educativas.

\section{LAS TRANSICIONES EDUCATIVAS, ¿OPORTUNIDADES O RETOS?}

Según la OCDE (Organisation for Economic Co-operation and Development, 2006), las transiciones son generalmente un estímulo para el crecimiento y el desarrollo, pero si resultan demasiado abruptas y se manejan sin cuidado tienden a suponer un riesgo de regresión y fracaso. Con el paso del tiempo se ha comprobado que estas situaciones generan en el alumnado cierta desorientación si se dan de forma rápida (antes de que puedan interiorizarse los cambios), lo que constituye una situación de riesgo (Spinath y Spinath, 2005). Precisamente, el comienzo de la escolaridad obligatoria ha sido definido por Dockett y Perry (2004a) como uno de los grandes retos en los primeros años de la infancia. Al hilo de esta cuestión, León (2009) afirma que los cambios que se producen en la vida se ven en parte influenciados por cómo ha teni- 
do lugar la experiencia de la primera transición. Fabian (2002) apunta en su trabajo que del éxito logrado en las transiciones depende, en gran medida, la huella que inevitablemente aparecerá en el futuro académico de la persona. Por tanto, puede afirmarse que las transiciones educativas influyen notablemente en la vida del alumnado, de ahí la importancia que tiene su tratamiento, revisión y estudio.

Numerosos expertos en la temática han concluido que las transiciones educativas representan momentos especialmente críticos. La experiencia de vivir la discontinuidad ante diversas circunstancias afecta al crecimiento personal, social y académico; y produce la asunción de sentimientos de pérdida de lo conocido, desasosiego, incertidumbre, nerviosismo, cambio de estatus y modificación del rol académico desempeñado por el alumnado dentro del aula (Sierra, 2017). Esta idea ha sido también refrendada por Alvarado y Suárez (2010), quienes consideran que las transiciones son momentos críticos que vive el alumnado cuando pasa de un ambiente educativo a otro y que, indudablemente, pueden afectar a su aprendizaje. En opinión de Gairín (2005), las transiciones siempre son problemáticas en la medida en que suponen una serie de cambios (normativos, docentes y de contexto) que influyen en los estratos psicológicos y sociales. Por su parte, Monarca, Rappoport y Sandoval (2013) confirman que las transiciones afectan de forma desigual a los estudiantes y pueden convertirse ya no tanto en una oportunidad como en un posible obstáculo para su desarrollo y aprendizaje. En términos de rendimiento pueden incluso constituirse en detonante de fracaso escolar. En ocasiones, se comprueba que el alumnado que se encuentra bajo los efectos de este proceso baja su rendimiento académico (Monarca, 2013).

Con respecto al alumnado que presenta mayor riesgo de exclusión o vulnerabilidad, igualmente hay que reconocer el impacto que tienen las transiciones (Azorín, 2019; Fabián y Dunlop, 2007). Los problemas derivados de la transición pueden llegar a retroalimentar una fuente desigual de oportunidades para el alumnado, de ahí que Petriwskyj (2014) y Azorín (2018a) aludan al carácter inclusivo que han de tener las transiciones escolares y la importancia de la búsqueda de acuerdos y actuaciones comunes sobre estas que salvaguarden el cumplimiento del principio de equidad. Asimismo, Arnup (2014) incide en la importancia de construir conexiones alrededor del proceso de transición mediante el impulso de alianzas y recursos para una mayor inclusión. 
Por otro lado, aunque el proceso de transición es cierto que conlleva momentos de cambio complejos en los que pueden surgir dificultades, también plantea nuevas oportunidades y expectativas. En última instancia, cuando la transición es percibida positivamente por parte del alumnado, hace que este desarrolle un sentido de pertenencia hacia el nuevo entorno, lo que le aporta cierta estabilidad y mejor predisposición en el proceso de enseñanza-aprendizaje (Dockett y Perry, 2004b; Fabian, 2007). En definitiva, que el alumnado sienta que forma parte del escenario por el que "transita" es lo que constituye la piedra angular de este proceso.

\section{La transición de Educación Infantil a Educación Primaria}

A medida que los niños y las niñas pasan de sus experiencias previas (preescolar, cuidado infantil, hogar, otros cuidados fuera del hogar) a la escuela, experimentan muchos cambios, entre ellos, la evolución de un enfoque pedagógico basado principalmente en el juego a una pedagogía más estructurada (Ballam, Perry y Garpelin, 2017; Chan, 2010). Con respecto a las transiciones educativas de la primera infancia, Peralta (2007) identifica la primera transición como aquella que se da en el escenario familiar (guardería o párvulos) y la segunda gran transición como aquella que modula del ambiente familiar-social a la institución externa (Educación Infantil), constituyendo la tercera transición el paso a la Educación Primaria.

El estudio de la transición de la etapa de Educación Infantil a la etapa de Educación Primaria es una línea de indagación importante y creciente que ha acaparado la atención de la investigación educativa en las últimas décadas (Argos y Ezquerra, 2004). Diferentes estudios han puesto de relieve que a lo largo de este proceso el alumnado ha de adaptarse a situaciones que le vienen impuestas y ajustarse a un nuevo entorno, en el que cambian los pares, el profesorado y la metodología, entre otros aspectos (Berlin, Dunning y Dodge, 2011).

En la etapa de Educación Infantil se trabaja el desarrollo de las competencias, las habilidades, las destrezas, las actitudes y las conductas que, a posteriori, serán puestas en práctica en Educación Primaria. Así pues, la estrecha coordinación e implicación activa del profesorado que imparte docencia en 
ambos tramos es fundamental. Cuando el profesorado de Educación Infantil de 5 años y el profesorado de $1 .^{\circ}$ de Educación Primaria (tanto los tutores y tutoras como los especialistas) trabajan de forma conjunta, ello redunda en un beneficio para el alumnado y su vivencia del cambio de etapa como un proceso ordenado, coherente, secuenciado, coordinado, planificado, de carácter progresivo y asentado sobre el principio de continuidad.

En consecuencia, Argos, Ezquerra y Castro (2011b) exponen que los planteamientos globalizadores que suelen estar firmemente instaurados en los contextos de Educación Infantil pierden gran parte de su protagonismo al adentrarse en la etapa de Educación Primaria. Como se ha descrito anteriormente, el paso de Infantil a Primaria implica una serie de cambios importantes para el alumnado: profesorado, espacios, distribución del tiempo, rutinas de clase, metodología de trabajo, materiales, recursos y evaluación son asuntos de peso que requieren atención. Todos estos aspectos afectan al alumnado y provocan en el profesorado una reflexión sobre los elementos organizativos, curriculares y metodológicos que han de tenerse en cuenta para la toma de acuerdos acerca de este proceso.

Por otro lado, el alumnado que vivencia esta situación en primera persona observa que: 1) cambia de profesorado y se reduce notablemente el tiempo de tutor con la entrada de los especialistas; 2) se incorpora a un espacio nuevo, con un aula que se organiza de manera distinta (en ocasiones, vienen de estar sentados por grupos a tener que hacerlo individualmente); 3) la metodología es diferente (deben prestar mayor atención y mantener silencio durante las explicaciones, aumenta el trabajo personal, se incide en el cuidado del material, en el desarrollo de hábitos de estudio independiente, en la consolidación del respeto a los adultos y en la adaptación a nuevas rutinas, como la lectura diaria y los deberes que se mandan para casa, evolucionando de una metodología con una gran carga lúdica y flexibilidad curricular a otra más formal y rígida); 4) acontecen modificaciones en los horarios y la distribución del tiempo de clase, y 5) se acentúa la rendición de cuentas con pruebas estandarizadas y exámenes que permiten comprobar la adquisición de los contenidos. Asimismo, cuando se produce la transición al inicio del primer trimestre, el alumnado de $1 .^{\circ}$ de Primaria mantiene las características y estructuras psicológicas propias del alumnado de Infantil de 5 años durante los primeros meses del curso escolar. Por ello, hay que tener en cuenta que la adaptación a todos 
estos cambios en algunos estudiantes puede durar unos meses y, en el caso de otros, prolongarse durante más tiempo.

Finalmente, Tamayo (2014) establece que dentro de la etapa de Educación Primaria se empieza a considerar el proceso de aprendizaje como una actividad más seria y responsable, pues es en este momento cuando se sientan las bases para futuros aprendizajes y se adquieren los hábitos y procedimientos de trabajo necesarios para afrontar con éxito el proceso de escolarización.

\section{La transición de Educación Primaria a Educación Secundaria}

La transición de Educación Primaria a Educación Secundaria ha sido extensamente documentada (Flores y Gómez, 2010; Monarca, 2013; Monarca y Rincón, 2010; Monarca et al., 2013; Lorenzo, 2010; Sierra y Parrilla, 2014). A este respecto, Sebastián (2015) analiza e interpreta en su investigación los problemas pedagógicos y psicosociales a los que hacen frente los estudiantes que son abocados a una ruptura de su vida escolar pasada y la antesala de otros tránsitos en su vida futura. Este autor concluye en su trabajo que las consecuencias que se derivan del paso de Primaria a Secundaria son las siguientes: 1) cambia el clima institucional (tipo de centro, normas de convivencia, exigencia de autorregulación); 2) las relaciones con el profesorado son más lejanas e impersonales; 3) aumenta el número de docentes por grupo y la exigencia hacia el alumnado no tiene un carácter global como en Primaria, sino que se compartimenta, y 4) la metodología es distinta no solo entre las etapas, sino también entre los profesores que forman el equipo educativo de un curso. Unido a lo anterior, la transición de Primaria a Secundaria coincide además con la adolescencia, motivo que se suma a lo expuesto con anterioridad sobre el cambio de centro educativo y de cultura escolar.

La adaptación del alumnado a la etapa de Secundaria ha de tener en cuenta la nueva cultura individualista, centrada en las disciplinas y orientada a los resultados. En la Educación Secundaria Obligatoria (ESO), las aulas son más numerosas, las metodologías son menos participativas y cambian las formas de acometer el proceso de enseñanza-aprendizaje. En síntesis, hay que reconocer la existencia de nuevos ritmos y estilos de trabajo, un menor control por parte del profesorado y una mayor libertad organizativa del alumnado, 
junto con los cambios que vienen motivados por procesos de evaluación más exigentes.

De hecho, por lo general, las notas de $1 .^{\circ}$ de ESO son inferiores a las de 6. ${ }^{\circ}$ de Educación Primaria. Según Monarca y Rincón (2010), la obtención de notas demasiado bajas y una gran cantidad de suspensos forma parte de una realidad que suele repetirse en $1 .^{\circ}$ de ESO, debido a las discontinuidades apreciadas durante la transición de Educación Primaria a Educación Secundaria en aspectos tales como la metodología de las clases, las relaciones con el profesorado o el tipo de evaluación. Rupturas para las que el alumnado a veces no está preparado y que podrían explicar en cierto modo los altos índices de fracaso escolar.

Desde el punto de vista de la inclusión educativa, se ha demostrado que para un porcentaje relativamente significativo de estudiantes de primer año de Educación Secundaria ciertas características relacionadas con las prácticas docentes, junto con otras de la cultura de los centros, pueden suponer el inicio o el refuerzo de los procesos de exclusión educativa (Monarca et al., 2013).

En el caso que nos ocupa, con el cambio de Educación Primaria a Educación Secundaria, Lorenzo (2010) explica que el alumnado procede de un centro escolar en el que ha compartido nueve años de su vida (tres como estudiante de la etapa de Educación Infantil y seis como estudiante de Educación Primaria), con el mismo grupo de docentes, compañeros y compañeras, instalaciones, normas de convivencia y horarios a una nueva cultura educativa con plantilla docente distinta, otros pares, instalaciones, distribución temporal de materias... en definitiva, una nueva organización escolar. A esto Flores y Gómez (2010) añaden el aumento de la carga de trabajo, una mayor demanda de autonomía e independencia al acometer tareas tanto dentro como fuera del aula, la modificación de las formas de evaluación y la falta de apoyos para responder a estos cambios.

En síntesis, Gimeno (1996) afirma que esta ruptura de contexto y de ambiente a la que nos venimos refiriendo exige un nuevo posicionamiento en el que el alumnado pasa de un grupo en el que quizás se muestra más responsable y popular a otro desconocido de pares y docentes, en el que se enfrenta a una evaluación más severa. Esta situación plantea a los centros educativos el reto de introducir medidas educativas que contribuyan a la reflexión, actualización y puesta en práctica de estrategias tendentes a favorecer una transi- 
ción progresiva y positiva, de manera que se produzca una adaptación en el menor tiempo posible y en las mejores condiciones (Tamayo, 2014). En esta dirección, hay que establecer nexos de unión para la continuidad entre etapas educativas, lo que facilitará mejores transiciones educativas y promoverá la adquisición de un desarrollo integral, ajustado y equilibrado.

\section{Hacia un ENFOQue COMUNITARIO}

Las transiciones son consubstanciales a la persona, todos pasan por ellas, suponen cambios, son procesuales y no se vivencian en solitud, sino que tienen una proyección de naturaleza social (Rodríguez, 2016). En esta línea, la Organización de los Estados Americanos (2009) alude a una responsabilidad compartida entre el niño o la niña, los pares, la familia, el profesorado y el entorno, lo que deriva en la adopción de una dimensión comunitaria de la transición. Para Fiuza y Sierra (2014), las transiciones educativas son procesos que implican a toda la comunidad (especialmente al alumnado, al profesorado y a las familias) y deben ser abordados de manera conjunta para propiciar una continuidad armónica entre todas las etapas educativas. De igual modo, Argos et al. (2011b) entienden que para favorecer esta continuidad tienen que instaurarse procesos de articulación que permitan guiar sin sobresaltos y de manera continua el proceso de enseñanza-aprendizaje.

Kraft-Sayre y Pianta (2000) hace ya unos años utilizaron un modelo ecológico-dinámico para explicar la transición de preescolar a la escuela, mediante la responsabilidad compartida del conjunto de individuos que hay alrededor del alumnado, y la naturaleza dinámica de las relaciones entre estos agentes (pares, familia, profesorado, comunidad), que influyen determinantemente en la infancia. Siguiendo este argumento, Dunlop (2014) concibe la transición como sistema ecológico, modelo que actualmente se encuentra en plena efervescencia (figura 1).

De forma paralela, Antúnez (2007) ha explorado las transiciones educativas con un particular énfasis en las articulaciones entre escuela, familia y comunidad, mientras que Parrilla y Sierra (2015) han destacado la concepción en clave comunitaria que caracteriza al proceso de transición. Asimismo, los procesos de transición no pueden acotarse ni ceñirse exclusivamente al 
FIGURA 1

La transición como sistema ecológico

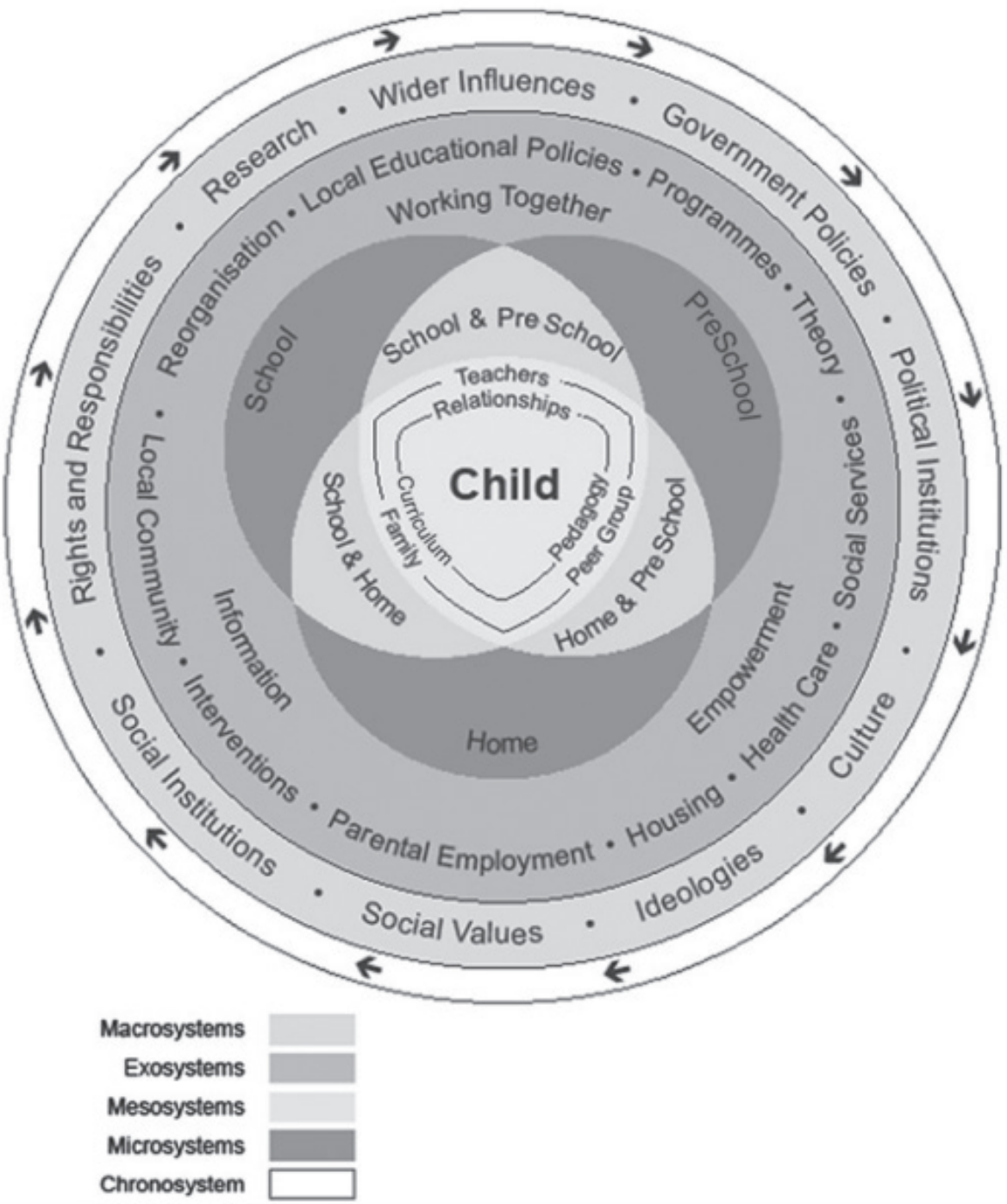

Fuente: Dunlop (2014: 42). 
alumnado que transita. Por el contrario, estos procesos deberían articularse adoptando un carácter dialógico y colaborativo, incluyendo las relaciones entre centros, profesorado y comunidad. Unido a ello, las transiciones han de sustentarse en estrategias planificadas que involucren el hogar, los profesionales y el centro educativo con la intencionalidad de garantizar la continuidad y que las necesidades del alumnado se cumplan adecuadamente (Bulkeley y Fabian, 2006; Smart, Sanson, Baxter, Edwards y Hayes, 2008).

Así pues, asumiendo la importancia que tiene la familia en las transiciones educativas, resulta necesario indicar que, como todo cambio, el paso a una nueva etapa educativa no resulta una tarea fácil. Un proceso de tal calibre precisa del apoyo y de la implicación activa de las familias (Isorna, Navia y Felpeto, 2013) y de su coordinación efectiva con el profesorado para conseguir una buena acomodación al nuevo periodo escolar. De igual modo, la literatura ha destacado el papel de la familia y del profesorado en la transición educativa como agentes facilitadores de esta (Hindman, Skibbe y Morrison, 2011). De hecho, dentro de las acciones comunes del profesorado que aborda la transición escolar se encuentran las actuaciones orientadas al trabajo con las familias y la preparación conjunta del proceso de adaptación a la nueva situación que esta circunstancia plantea por parte de los equipos directivos y docentes.

A su vez, para que las transiciones educativas puedan llevarse a cabo con éxito, se considera vital la colaboración y coordinación entre centros educativos, dado que, en el proceso de transición escolar, el principio de colaboración es fundamental. Precisamente, el estudio liderado por Hernández y Navarro (2018) presenta una red escolar pionera que ha sido constituida para que el profesorado trabaje de forma conjunta en el planteamiento de propuestas escolares que faciliten la transición del alumnado del nivel de Primaria al de Secundaria. En este sentido, la colaboración de los centros educativos que imparten enseñanzas consecutivas en una misma zona y que, a menudo, atienden a un grupo de estudiantes que provienen de un contexto similar puede aportar nuevas vías para la mejora de las transiciones. Sin embargo, hay que reconocer que, aunque la tendencia a colaborar entre centros educativos por cuestiones asociadas a las transiciones educativas empieza a ser incipiente, todavía queda mucho por hacer para que estas acciones se extiendan en la práctica. Estudios como el desarrollado por Azorín (2018b) 
indican que apenas se ponen en marcha actuaciones entre centros educativos de una misma área geográfica para coordinar aspectos relacionados con las transiciones escolares, hecho que da cuenta de la débil cultura de colaboración que persiste en el ámbito educativo.

Lo expresado anteriormente evidencia la existencia de fronteras en las transiciones educativas. A este respecto, Vogler et al. (2008) se refieren a la teoría del cruce de fronteras centrada en las transiciones que ocurren cotidianamente, una teoría sustentada en "dominios" (por ejemplo: la escuela), separados por "fronteras" o "límites" que los individuos diariamente deben cruzar a fin de interpretar los roles que les corresponden en cada dominio (Campbell Clark, 2000). Según afirman Dockett, Petriwskyj y Perry (2014), es posible considerar el espacio por donde transita el alumnado que ha dejado un contexto (preescolar) pero que aún no ha ingresado en otro (escuela) como zonas fronterizas que estos atraviesan. En síntesis, la influyente obra de Giroux (2005) titulada Border crossings (Cruces fronterizos) argumenta que la lucha sobre territorios, espacios y zonas de contacto es donde el poder opera para expandirse o para reducir la distancia y la conexión entre individuos, grupos y lugares.

Para terminar, dentro de esta visión holística cabría señalar al menos la influencia que ha tenido la pedagogía sistémica desarrollada por Bert Hellinger y el vínculo inherente del propio proceso de tránsito con sus constelaciones. En esta línea, la obra de Perellada y Traveset (2016) pone atención al viaje que se produce al transitar por caminos desconocidos, en una escuela y una sociedad que es diversa, que está abierta a trabajar en red y a potenciar el equilibrio mente-corazón que todo ser humano necesita adquirir, teniendo siempre como telón de fondo a la comunidad.

\section{Consideraciones finales}

Este último apartado pone el foco de atención en los retos pendientes que tiene la investigación actual en el campo de las transiciones educativas a la vista de los aspectos abordados en el artículo. En primer lugar, hay que reconocer que la recogida de la voz de todos los implicados en el proceso de transición (no exclusivamente del profesorado sino también del alumnado 
y de sus familias) es uno de los principales desafíos. El testimonio de los más pequeños puede dar una información privilegiada acerca de cómo viven este proceso. Las investigaciones sobre las transiciones educativas cada vez más recogen la voz del alumnado en calidad de protagonista indiscutible. En el estudio desarrollado a este respecto por Parrilla, Gallego y Sierra (2016) diversos estudiantes fueron invitados a dar y discutir ideas sobre las transiciones, y tuvieron asimismo la oportunidad de guiar y proponer formas de enfrentar problemas reales vividos por ellos en el proceso de transición. Atender y escuchar las voces de toda la comunidad educativa es un requerimiento de primer orden, hecho que incluye al alumnado y a sus familias, colectivos que tradicionalmente han sido silenciados haciendo uso de una pedagogía poco inclusiva. En definitiva, al incluir estas otras voces se obtiene una visión más completa de la realidad estudiada, reduciendo así posibles sesgos (Argos, Ezquerra y Castro, 2011a; Castro, Argos y Ezquerra, 2015; Castro et al., 2011; Castro, Ezquerra y Argos, 2012).

Por otro lado, Monarca y Rincón (2010) han sintetizado algunas propuestas de mejora para abordar las transiciones educativas, entre ellas: 1) un currículo integrado, con un enfoque más global de la enseñanza; 2) la asignación del profesorado de los primeros cursos de ESO, con capacidad de adaptación y comprensión hacia la etapa evolutiva en la que se produce este proceso; 3 ) una metodología variada, que responda a los distintos estilos de aprendizaje, a la par que activa, para que fomente la participación, que esté adaptada al grado de desarrollo del alumnado y que tenga en cuenta el nivel curricular, el desarrollo de competencias básicas en general, los ritmos de trabajo y un nivel de exigencia que aumente de forma progresiva; 4) experiencias de aprendizaje significativo y funcional, relacionando los contenidos previos con el mundo cotidiano; 5) medidas educativas específicas con el alumnado que repite curso, incluyendo cambios metodológicos y organizativos, y 6) utilizar los resultados de la evaluación para introducir las medidas de apoyo y refuerzo que sean necesarias, así como los ajustes metodológicos, de contenidos, organizativos, de acción tutorial, y las orientaciones a las familias que permitan prevenir posibles situaciones de fracaso escolar.

En esta misma dirección, Sebastián (2015) recoge entre sus propuestas de mejora: 1) el aumento de mecanismos de colaboración entre profesionales, de estos con las familias y entre instituciones educativas de una misma 
zona; 2) la incorporación de tutorías individualizadas, agrupamientos flexibles (máxime en las asignaturas instrumentales), trabajo de las normas de convivencia, identificación de comportamientos inadecuados y desarrollo de procedimientos de resolución de conflictos; 3) la coordinación de líneas metodológicas, capacidades transversales a diferentes áreas, continuidad de objetivos, contenidos y exigencias; 4) el acompañamiento del alumnado durante este proceso, y 5) la organización de seminarios de formación en centros y grupos de trabajo para los docentes de los diferentes niveles. Por su parte, $\mathrm{Pa}$ dilla (2009) expone que se debe reflexionar sobre los aspectos que garantizan una mejor transición, entre ellos: 1) el profesorado de Educación Primaria ha de conocer las características básicas del alumnado que finaliza la Educación Infantil; 2) las propuestas didácticas han de tener un formato único en ambas etapas elaborándose de forma coordinada por los correspondientes equipos docentes. Especial importancia cobra en este caso el hecho de que el método lecto-escritor sea común; 3) hay que maximizar la coherencia y conexión de las diferentes áreas y ámbitos de experiencia; 4) debe existir continuidad en los principios metodológicos, las actividades, materiales, recursos, agrupamientos y distribución de tiempos y espacios, y 5) se han de realizar actuaciones conjuntas para que el alumnado vaya conociendo los nuevos entornos, así como al profesorado y a los pares de referencia.

En suma, puede concluirse que el paso de una etapa educativa a otra precisa de una serie de iniciativas encaminadas a minimizar los saltos o dificultades que puedan derivarse de los aspectos organizativos y curriculares. A este respecto, el equipo directivo y docente de los centros escolares ha de comprometerse a ejercer un papel facilitador y orientador durante este período para que los cambios que se produzcan no afecten negativamente en la evolución personal e integración socioeducativa del alumnado. De igual forma, el apoyo familiar es de gran ayuda en este proceso, de ahí que se abogue por una relación familia-escuela lo más cercana y efectiva posible.

Con respecto a las limitaciones por las que atraviesa la investigación sobre las transiciones educativas, Dockett et al. (2014) concluyen lo siguiente: 1) confusión en torno a los términos orientación y transición; 2) familias, servicios y educadores que carecen de información suficiente, así como de recursos para orientar sus prácticas y atender a la diversidad del alumnado; 3) una planificación de la transición centrada mayoritariamente en las escue- 
las individuales, en lugar de la comunidad en general, y 4) tendencia a pasar por alto el compromiso con otros profesionales y servicios, obviando de este modo la relevancia que tiene el enfoque comunitario al que se ha hecho alusión a lo largo del texto.

En relación con las propias limitaciones o lagunas que presenta el artículo, cabría preguntarse si las transiciones acaban donde finaliza la atención de este documento. Debería señalarse en este sentido que, como ya adelantaba el marco teórico en lo que al carácter permanente de este fenómeno se refiere, más allá de las transiciones comentadas, trabajos como el desarrollado por Ezquerra, Argos, Fernández-Salinero y González-Geraldo (2016) profundizan en las transiciones formativas para una práctica docente democrática con especial incidencia en la dialéctica entre los contextos universitario y escolar.

Finalmente, este apartado se hace eco a modo de prospectiva de la influyente obra Transitions to School. International Research, Policy and Practice, en la que Dockett et al. (2014) concluyen las direcciones emergentes que está tomando la investigación actual en el campo de las transiciones, y que en cierta medida se han concretado de forma pormenorizada en este trabajo, destacando, particularmente: 1) la necesidad de explorar más a fondo el papel y la contribución de la política educativa en materia de transición en la escuela; 2) la incorporación de las voces de todas las partes interesadas en la transición, con especial énfasis en la perspectiva y voz del alumnado y de sus familias; 3) el reconocimiento del papel que pueden desempeñar las alianzas efectivas en las distintas transiciones, y 4) la exploración del impacto que tienen, tanto a corto como a largo plazo, las transiciones educativas en la vida de las personas.

\section{BIBLIOGRAFÍA}

Alvarado, S. V. y SuÁrez, M. C. (2010). Las transiciones escolares: una oportunidad de desarrollo integral para niños y niñas. Revista Infancias Imágenes, 9(1), 70-84.

Álvarez, E. y Rodríguez, Y. J. (2018). La transición educativa del centro de desarrollo infantil al grado preescolar en Caucasia, Antioquia: ¿un proceso o un paso? Infancias Imágenes, 17(1), 67-77. 
Anderson, L., Jacobs, J., Schramm, S., y Splittgerber, F. (2000). School transitions: beginning of the end or a new beginning? International Journal of Educational Research, 33, 325-339.

Antúnez, S. (2007). La transición entre etapas. Barcelona: Graó.

Argos, J. y Ezquerra, M. P. (2004). Tan lejos, tan cerca. La continuidad entre la escuela infantil y la primaria. En M. D. García y V. Marín (coords.), La educación infantil y la formación del profesorado hacia el siglo XXI: integración e identidad (pp. 359-369). Córdoba: Servicio de Publicaciones de la Universidad de Córdoba.

Argos, J., Ezquerra, M. P. y Castro, A. (2011a). Escuchando la voz de la infancia en los procesos de cambio e investigación educativos. Aproximación al estudio de las transiciones entre las etapas de educación infantil y educación primaria. Revista Iberoamericana de Educación, 54(5), 1-18.

Argos, J., Ezquerra, M. P. y CAstro, A. (2011b). Metáforas de la transición: la relación entre la escuela infantil y la escuela primaria y la perspectiva de futuros docentes en educación infantil. Education XX1, 14(1), 135-156. Arnup, M. (2014). Building Connections Around Transition: Partnerships and Resources for Inclusion. En B. Perry, S. Dockett y A. Petriwskyj (eds.), Transitons to School International Research, Policy and Practice (pp. 261-276). Australia: Springer.

Azorín, C. (2018a). Abriendo fronteras para la inclusión: la Ecología de la Equidad. Revista de Educación Inclusiva, 11(1), 213-228.

Azorín, C. (2018b). Percepciones docentes sobre la atención a la diversidad: propuestas desde la práctica para la mejora de la inclusión educativa. ENSAYOS, Revista de la Facultad de Educación de Albacete, 33(1), 173-188. Azorín, C. (2019). Diseño y evaluación de un plan para la atención del alumnado inmigrante con desconocimiento del español. Contextos Educativos, 23, 179-197.

Azorín, C. y Ainscow, M. (en prensa). Guiding schools on their journey towards inclusion. International Journal of Inclusive Education.

Ballam, N.; Perry, B. y Garpelin, A. (2017). International Perspectives on the Pedagogies of Educational Transitions. En N. Ballam, B. Perry y A. Garpelin (eds.), Pedagogies of Educational Transitions. International Perspectives on Early Childhood Education and Development (pp. 1-12). Cham: Springer. 
Bereziartua, J.; Intxausti, N. y Odriozola, A. (2017). Acción tutorial en la transición del alumnado de las escuelas del medio rural a la educación secundaria. Tendencias pedagógicas, 29, 189-210.

Berlin, L.; Dunning, R. y Dodge, K. (2011). Enhancing the Transition to Kindergarten: A randomized trial to test the Efficacy of the 'Stars' summer kindergarten orientation program. Early Childhood Research Quarterly, 26(2), 247-254.

Bulkeley, J. y Fabian, H. (2006). Well-being and Belonging during Early Educational Transitions. International Journal of Transitions in Childhood, 2, 18-30.

CAmpbell Clark, S. (2000). Work/family border theory: A new theory of work/family balance. Human Relations, 53(6), 747-770.

Castro, A., Argos, J. y Ezquerra, P. (2015). La mirada infantil sobre el proceso de transición escolar desde la etapa de educación infantil a la de educación primaria. Perfiles Educativos, XXXVII(148), 34-49.

Castro, A.; Ezquerra, P. y Argos, J. (2012). La transición entre la Escuela de Educación Infantil y la de Educación Primaria: perspectivas de niños, familias y profesorado. Revista Española de Pedagogía, 253, 537-552.

Castro, A.; Ezquerra, P. y Argos, J. (2018). Profundizando en la transición entre educación infantil y educación primaria: la perspectiva de familias y profesorado. Teoría de la Educación. Revista Interuniversitaria, 30(1), 217-240.

Castro, M.; Díaz, M.; Fonseca, H.; León, A.T.; Ruíz, L. S. y Umaña, W. (2011). Las relaciones interpersonales en la transición de los estudiantes de la primaria a la secundaria. Revista Electrónica Educare, 15(1), 193-210.

Chan, W. L. (2010). The Transition from Kindergarten to Primary School, as Experienced by Teachers, Parents and Children in Hong Kong. Early Child Development and Care, 180(7), 973-993.

Corominas, E. e Isus, S. (1998). Transiciones y orientación. Revista de Investigación Educativa, 16(2), 155-184.

Dockett, S. y Perry, B. (2004a). Starting school: Perspectives of Australian children, parents and educators. Journal of Early Childhood Research, 2(2), 171-189. 
Dockett, S. y Perry, B. (2004b). What makes a successful transition to school? Views of Australian parents and teachers. International Journal of Early Years Education, 12(3), 217-230.

Dockett, S. y Perry, B. (2007). Transitions to school: Perceptions, expectations, experiences. Sydney: University of NSW Press.

Dockett, S. y Perry, B. (2013). Trends and tensions: Australian and international research about starting school. International Journal of Early Years Education, 21(2-3), 163-177.

Dockett, S., Petriwskyj, A. y Perry, B. (2014). Theorising Transition: Shifts and Tensions. En B. Perry, S. Docket y A. Petriwskyj (eds.), Transitons to School International Research, Policy and Practice (pp. 1-18). Australia: Springer.

Dunlop, A.W. (2014). Thinking About Transitions: One Framework or Many? Populating the Theoretical Model Over Time. En B. Perry, S. Dockett y A. Petriwskyj (eds.), Transitons to School International Research, Policy and Practice (pp. 31-46). Australia: Springer.

Elias, M. y DazA, L. (2017). ¿Cómo deciden los jóvenes la transición a la educación postobligatoria? Diferencias entre centros públicos y privadosconcertados. Revista de la Asociación de Sociología de la Educación, 10(1), 5-22.

Ezquerra, P., Argos, J., Fernández-Salinero, C. y González-Geraldo, J. L. (2016). Transiciones formativas para una práctica docente democrática: la dialéctica entre los contextos universitario y escolar. En I. Carrillo (coord.), Democracia y Educación en la formación docente (pp. 261-289). Eumográfic: Servei de Publicacions de la Universitat de Vic.

Fabian, H. (2002). Empowering children for transitions. En H. Fabian y A.W. Dunlop (eds.), Transitions in the early years (pp. 123-134). London: Routledge.

Fabian, H. (2007). Informing transitions. En A.W. Dunlop y H. Fabian (eds.), Informing transitions in the early years (pp. 3-17). Maidenhead: Open University Press.

FABIAN, H. y Dunlop, A. (2007). Outcomes of good practice in transition processes for children entering primary school. La Haya: Bernard van Leer Foundation. 
Fernández, J. y SAntos-Bocero, G. L. (2014). Orientar las transiciones del alumnado inmigrante: más que un reto multiprofesional. Revista Española de Orientación y Psicopedagogía, 25(2), 8-23.

Finnish Ministry of Education and Culture (2017). Finnish Country Note on Transitions in ECEC. Review of Policies and Practices for Transitions from Early Childhood Education and Care to Primary Education. Finlandia: Ministry of Education and Culture.

FiuzA, M. J. y Sierra, S. (2014). Un camino para facilitar las transiciones educativas. International Journal of Developmental and Educational Psychology, 3(1), 109-118.

Flores, R. y Gómez, J. (2010). Un estudio sobre la motivación hacia la escuela secundaria en estudiantes mexicanos. Revista Electrónica de Investigación Educativa, 12(1), 1-21.

Gairín, J. (2005). El reto de la transición entre etapas educativas. Aula de Innovación Educativa, 142, 12-17.

Gimeno, J. (1996). La transición a la educación secundaria. Madrid: Morata.

Giroux, H. A. (2005). Border crossings. Nueva York: Routledge.

Hernández, E. y Navarro, M. J. (2018). La participación en redes escolares para promover la mejora educativa, un estudio de caso. Profesorado. Revista de currículum y formación del profesorado, 22(2), 71-90.

Hindman, A., Skibbe, L. y Morrison, F. (2011). An Examination on Teacher's Practices and their Unique Contributions to Children's Early Academic Outcomes. En D. Laverick y M. Renck (eds.), Transitions to Early Care and Education. International perspectives on making schools ready for young children (pp. 57-73). Nueva York: Springer.

Isorna, M.; NAvia, C. y Felpeto, M. (2013). La transición de la educación primaria a la educación secundaria: sugerencias para padres. Innovación Educativa, 23, 161-177.

Kraft-SAYRe, M. y PiAnTA, R. (2000). Enhancing the transition to kindergarten: Linking children, families, and schools. Charlottesville: University of Virginia.

LEÓN, S. (2009). ¿Por qué es necesario el periodo de adaptación en la Educación Infantil? Innovación y Experiencias educativas, 15, 1-9.

Lorenzo, F. (2010). Enfoque orientativo en la transición de primaria a secundaria. Autodidacta. Revista de la Educación en Extremadura, 9, 121-127. 
Michavila, F. y Esteve, F. (2011). La llegada a la universidad: ¿oportunidad o amenaza? CEE Participación Educativa, 17, 69-85.

Monaheng, M. (2017). Transición educativa de estudiantes con discapacidad: más allá de la escuela. ¿Qué pasará con él cuando yo no esté viva? Revista Colombiana de Educación, 73, 59-76.

Monarca, H. (2013). Aportes de la investigación sobre transiciones escolares a la orientación educativa en ESO. Revista Española de Orientación y Psicopedagogía, 24, 116-125.

Monarca, H.; Rappoport, S. y Sanvodal, M. (2013). La configuración de los procesos de inclusión y exclusión educativa. Una lectura desde la transición entre Educación Primaria y Educación Secundaria. Revista de Investigación en Educación, 11(3), 192-206.

MonarCa, H. y Rincón, J. (2010). Tránsito a la ESO ¿continuidad o ruptura? Cuadernos de Pedagogía, 401, 28-31.

Organisation for Economic Co-Operation And Development (2006). Starting strong II: Early childhood education and care. París: OECD.

OrganizACIÓN DE Los Estados AmERICANOs (2009). Las transiciones en la primera infancia: una mirada internacional. Washington: OEA.

PAdilla, R. (2009). Desarrollo psicoevolutivo en niños 6-12 años. Innovación y Experiencias Educativas, 14, 1-10.

Parrilla, A.; Gallego, C. y Sierra, S. (2016). When educational transitions are supported by students. Journal of Research in Special Education Needs, 16(1), 1104-1107.

Parrilla, M. A. y Sierra, S. (2015). Construyendo una investigación inclusiva en torno a las distintas transiciones educativas. Revista Electrónica Interuniversitaria de Formación del Profesorado, 18(1), 161-175.

Peralta, M. (2007). Estado del arte sobre pedagogía de la primera infancia (0 a 3 años) en Latinoamérica y el Caribe. Recuperado de: https://www .oei.es/historico/inicial/articulos/. Consulta: 1 de agosto de 2018.

Perellada, C. y Traveset, M. (2016). Las redes sutiles de la educación: Las ideas clave de la pedagogía sistémica. Barcelona: Octaedro.

Perry, B.; Dockett, S. y Petriwskyj, A. (2014). Transitons to School International Research, Policy and Practice. Australia: Springer. 
Petriwskyj, A. (2014). Critical Theory and Inclusive Transitions to School. En B. Perry, S. Dockett y A. Petriwskyj (eds.), Transitions to School International Research, Policy and Practice (pp. 201-215). Australia: Springer.

Petriwskyj, A.; Thorpe, K. y Tayler, C. (2005). Trends in construction of transition to school in three western regions, 1990-2004. International Journal of Early Years Education, 13(1), 55-69.

Rodríguez, F. M. (2016). Transición de Primaria a Secundaria: Factores de éxito. Tesis doctoral. Universidad de Barcelona.

Rogoff, B. (2003). The cultural nature of human development. Oxford: Oxford University Press.

SÁnchez, F. J. y Moreno, A. (2018). La transición escolar en España: un análisis de desigualdad y diferenciación. Revista del Centro Reina Sofía sobre Adolescencia y Juventud, 8, 68-83.

SEBAstián, V. (2015). Una reflexión sobre las transiciones educativas. De primaria a secundaria itraspaso o acompañamiento? Edetania, 48, 159-183.

Sierra, S. (2017). Hacia una Pedagogía de la Transición: Caminando de Infantil a Primaria. Tesis doctoral. Universidad de Vigo.

Sierra, S. y Parrilla, M. A. (2014). ¿Una oportunidad igual para todos? Investigando la transición a la educación secundaria en una comunidad socio-educativa. Revista Latinoamericana de Educación Inclusiva, 8(2), 85-102.

Skouteris, H.; Watson, B. y Lum, J. (2012). Preschool children's transition to formal schooling: The importance of collaboration between teachers, parents and children. Australasian Journal of Early Childhood, 37(4), 78-85.

Smart, D.; Sanson, A.; BaXter, B.; Edwards, B. y Hayes, A. (2008). Hometo-school transitions for financially disadvantaged children: Summary report. Sidney: The Smith Family and Australian Institute of Family Studies.

Solís, P. (2018). La transición de la secundaria a la educación media superior en México: el difícil camino a la cobertura universal. Perfiles Educativos, XL(159), 66-89.

Spinath, B. y Spinath, F. M. (2005). Longitudinal analysis of the link between learning motivation and competence beliefs among elementary school children. Learning and Instruction, 15, 87-102. 
TAMAYo, S. (2014). La transición entre etapas educativas: de Educación Infantil a Educación Primaria. Participación Educativa, 3(5), 131-137.

UnICEF (2012). School readiness: A conceptual framework. Nueva York: Unicef.

VAlls, G. (2003). La transición de Primaria a Secundaria. Cuadernos de Pedagogía, 327, 64-66.

Vogler, P.; Crivello, G. y Woodhead, M. (2008). La investigación sobre las transiciones en la primera infancia: Análisis de nociones, teorías y prácticas. Países Bajos: Fundación Bernard van Leer. 